\title{
Paramphistomidae (Trematoda: Digenea) de peixes \\ de água doce: dois novos gêneros da Colômbia e uma redescrição de Dadaytrema oxycephala (Diesing, 1836) Travassos, 1934, da Amazônia
}

\author{
Vernon E. Thatcher $\left[^{*}\right]$
}

\begin{abstract}
Resumo
Dois novos gêneros e espécies de Paramphistomidae Fischoeder, 1901 (Trematoda: Digenea) dos tratos intestinais de peixes foram descritos, a saber: Colocladorchis ventrastomis $\mathrm{n}$, gen, $\mathrm{n}, \mathrm{sp}$ de Prochilodus reticulatus; e Dadaytremoiaes grandistomis n. gen., n. sp. de Astyanax fasciatus e Chaetostomus leucomelas. Estas espécies foram co. letadas perto a Cali, Valle, Colômbia. Adicionalmente, o gênero Dadytrema foi redefinido, e D. oxy. cephala (Diesing, 1836) Travassos, 1934, de Colosso. ma bidens, procedente de Manaus, Amazonas, Brasil, foi redescrito.
\end{abstract}

\section{INTRODUÇĀO}

A familia Paramphistomidae Fischoeder 1901, é um grupo primitivo de trematódeos digenéticos com muitos representantes em répteis, pássaros e mamíferos, sendo poucos os gêneros conhecidos de peixes. A família é considerada como importante devido ao fato de infectar com freqüência os tratos digestivos de herbívoros domésticos e até, às vezes, o ser humano.

Entre as principais monografias sobre este grupo de helmintos, destacam-se as de Travassos (1934), Näsmark (1937) e Skrjabin (1949). Trabalhos gerais, que também tratam da familia são os de Yamaguti $(1953,1958,1971)$. As publicações de Willey $(1930,1933)$ ajudaram a clarificar certos pontos sobre a morfologia, e o estudo de Bennett (1936) segue sendo a melhor e mais detalhada explicaçăo dum ciclo biológico nesse grupo. Padilla (1978) avançou notavelmente nossos conhecimentos da morfologia e, filogenia da superfamilia Paramphistomoidea com sua caracterização da família Zonocotylidae Padilla, 1978, considerada como um grupo mais primitivo ainda que a Paramphistomidae.

(*) - Instituto Nacional de Pesquisas da Amazônia, Manaus
O presente estudo foi iniciado como um levantamento geral dos parasitas de peixes nos altos do rio Cauca, Valle, Colômbia, perto da cidade de Cali. O trabalho está sendo continuado na Bacia Amazônica, dando assim a oportunidade de comparar a helmintofauna de peixes das duas regiōes.

\section{MÉTODOS E MATERIAIS}

$\mathrm{Na}$ Colômbia os peixes foram comprados dos pescadores locais, e em Manaus, Amazonas, os peixes foram capturados com redes pelos funcionários da Divisão de Peixe e Pesca do Instituto Nacional de Pesquisas da Amazônia. Os métodos de necropsiar os hospedeiros e preparar os parasitas foram os mesmos usados anteriormente (Thatcher, 1978), mas com os vermes maiores foi preciso utilizar glicerina ou fenol para sua clarificação. Exemplares vivos também foram estudados. Os desenhos foram feitos com um microscópio de projeção. As medições foram feitas com ocular micrométrica, são dadas com os limites inferior e superior, e a média é indicada entre parênteses. Todas as medidas são dadas em milimetros exceto as dos ovos, miracídios e papilas que são em micra.

\section{SeÇȦo Sistemática}

\section{Colocladorchis n. gen.}

Diagnose Genérica: Paramphistomidae, Pseudocladorchiinee, com as características da família e subfamília. Corpo achatado, arredondado posteriormente, apontado anteriormente. Cutícula lisa. Ventosa oral arredonda$\mathrm{da}$, com divertículos pequenos dentro das 
paredes. Esôfago longo; bulbo esofagiano presente. Cecos largos, curtos, atingindo região equatorial. Acetábulo grande pósteroventral. Poro genital mediano, a nível da bifurcação intestinal. Testículos fracamente lobados, paralelos e ventro-laterais aos cecos, no terço mediano do corpo. Bolsa do cirro e cirro presentes. Ovário esférico, intra-testicular. Glândulas vitelínicas constituídas por duas massas irregulares, póstero-dorsais aos testículos. Útero ocupando espaço entre ovário e acetábulo. Sistema circulatório presente. Vesícula excretora em forma de bolsa, poro dorsal. Ovovivíparos. Parasitas intesti nais de peixes de água doce. Espécie tipo: C. ventrastomis $\mathrm{n}$. $\mathrm{sp}$.

\section{Colocladorchis ventrastomis $\mathrm{n} . \mathrm{sp}$. (Fig. 1)}

Hospedeiro: Prochilodus reticulatus Steindachner. Habitat: Intestino.

Procedência: Rios Media Canoa, Fraile e Jamundi, Colômbia (Valle).

Holótipo: Instituto Nacional de Pesquisas da Amazônia (INPA).

Parátipos: INPA e Museu de Zoologia da Universi dade de São Paulo.

Diagnose específica (baseada em 10 exemplares): Com as características do gênero. Corpo medindo $0,71-1,53(1,1)$ de comprimento e $0,40-0,89(0,57)$ de largura. Ventosa oral com $0,11-0,19 \quad(0,14)$ de diâmetro. Esôfago longo. Bulbo esofagiano medindo $0,08-0,11(0,09)$ de comprimento. Cecos estendendo-se até $0,52-0,86(0,67)$ da extremidade anterior. Acetábulo póstero-ventral, medindo $0,30-0,58(0,41)$ de diâmetro. Testículos mais compridos que largos, medindo $0,11-0,22$ $(0,17)$ de comprimento e $0,094-0,14(0,12)$ de largura. Pequena bolsa de cirro contendo cirro e células prostáticas presente; medindo $0,072-0,12(0,09)$ de comprimento e $0,060-0,096$ $(0,078)$ de largura. Ovário esférico, medindo $0,050-0,13(0,08)$ de diâmetro. Útero ocupando espaço entre ovário e acetábulo, pode estender-se dorsalmente ao acetábulo. Ovovivíparos. Ovos medem $36-49$ por $60-66$ (43 x 63) $\mu$. Miracídios intrauterinos medem $53-95$ por $85-142(69 \times 118) \mu$.

\section{DISCUSSÃo}

Colocladorchis ventrastomis n. gen., n. sp. aproxima-se mais do gênero Pseudocladorchis Daday, 1907, e da espécie tipo do mesmo, o P. cylindricus (Diesing, 1836), pela forma da ventosa oral, com pequenos divertículos dentro das paredes e pela posição dos testículos. A nova forma distingue-se de $P$. cylindricus em ter o ovário inter-testicular, as glândulas vitelínicas anteriores, compactas e um acetábulo maior e ventral em posiçăo. A nova forma não tem papilas circundando a boca e é menor em tamanho. Além disso, o novo gênero procedeu de um hospedeiro diferente $\mathrm{e}$ de outro sistema de rios.

O nome genérico indica o país de orıgem do material, e o nome específico faz referência à posição ventral do acetábulo.

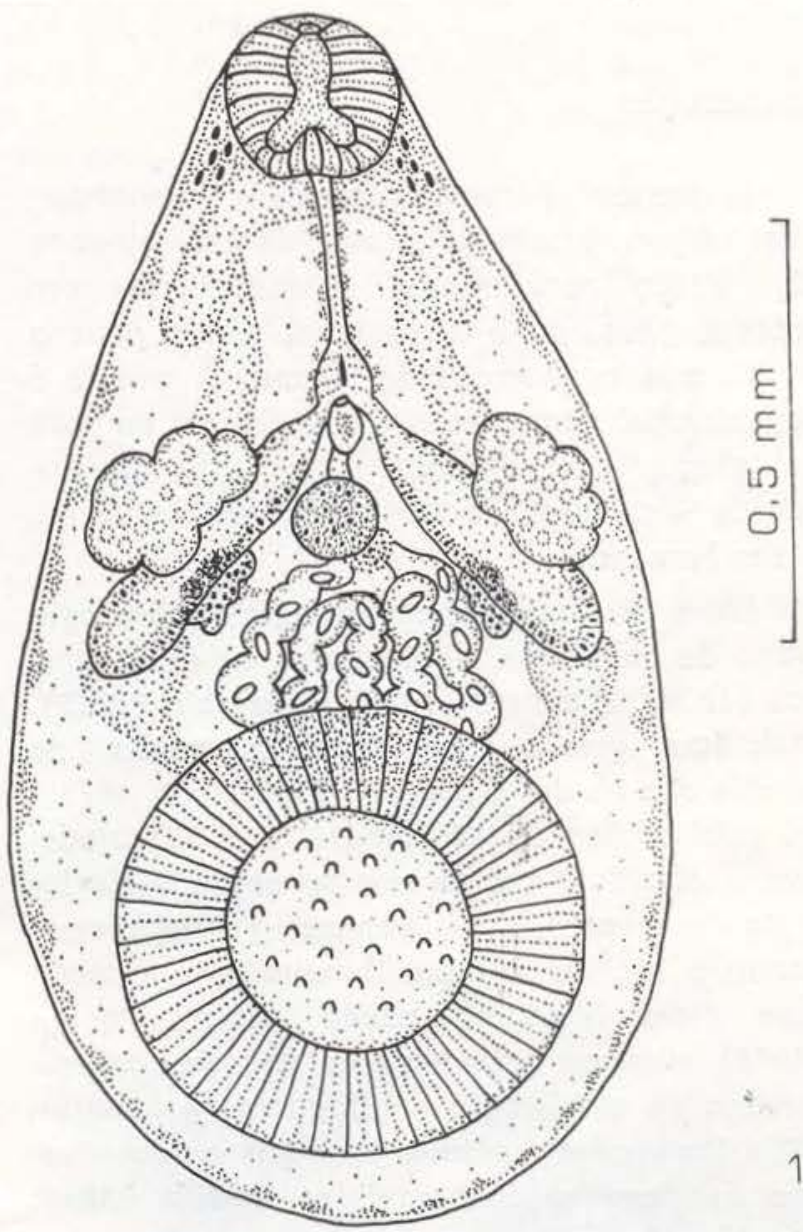

Fig. 1-Colocladorchis ventrastomis n. gen., n. sp. vista ventral. 
Diagnose Genérica: Paramphistomidae, Dadaytrematinae; com as características da Familia e Subfamília. Corpo alongado, cilíndrico. Cutícula lisa. Ventosa oral piriforme, com divertículos prominentes projectando posteriormente. Boca terminal, circundada por pequenas papilas, e com vários círculos de papilas posterior à boca. Esôfago longo com pequeno bulbo esofagiano. Cecos longos, sinuosos, terminando perto ao acetábulo. Acetábuio subterminal. Poro genital mediano e pos-bifurcal; com ventosa genital. Testículos profundamente lobados, pre-equatoriais, intercecaıs e invadindo as áreas cecais. Bolsa ge nital contindo vesícula seminal presente. Cirro ausente. Ovário esférico, no último quarto do corpo. Canal de Laurer presente. Glândulas vitelínicas constituídas por poucos folículos pequenos, laterais ao ovário e dorsais aos cecos. Útero ocupa espaço intercecal entre ovário e testículos. Sistema circulatório de tubos longitudinais associados com os cecos e com dois seios anteriores e dois seios posteriores, associados com as ventosas. Vesicula excretora em forma de bolsa. Poro dorsal. Ovoviviparos. Parasitas de peixes de água doce. Espécie tipo: D. oxycephala (Diesing, 1836) Travassos 1931.

Dadaytrema oxycephala (Diesing, 1836) Travassos, 1931.

(Fig. 2)

Hospedeiro: Colossoma bidens (L.) "Pirapitinga". Habitat: Intestino.

Procedência: Lago Janauacá, Manaus, Amazonas, Brasil.

Lâminas: Instituto Nacional de Pesquisas da Amazônia.

Diagnose específica (baseada em 10 exemplares): com as características do gênero. Corpo medindo $3,0-5,0 \quad(3,7)$ de comprimento e $1,1-1,3(1,1)$ de largura. Ventosa oral com $0,15-0,25 \quad(0,19)$ de comprimento e $0,09-0,18(0,13)$ de largura. Divertículos orais medindo $0,18-0,22 \quad(0,19)$ de comprimento e $0,14-0,18(0,16)$ de largura. Boca terminal, circundada de papilas com 4-5 $\mu$ de comprimento, e com quatro a cinco círculos de papilas pos-

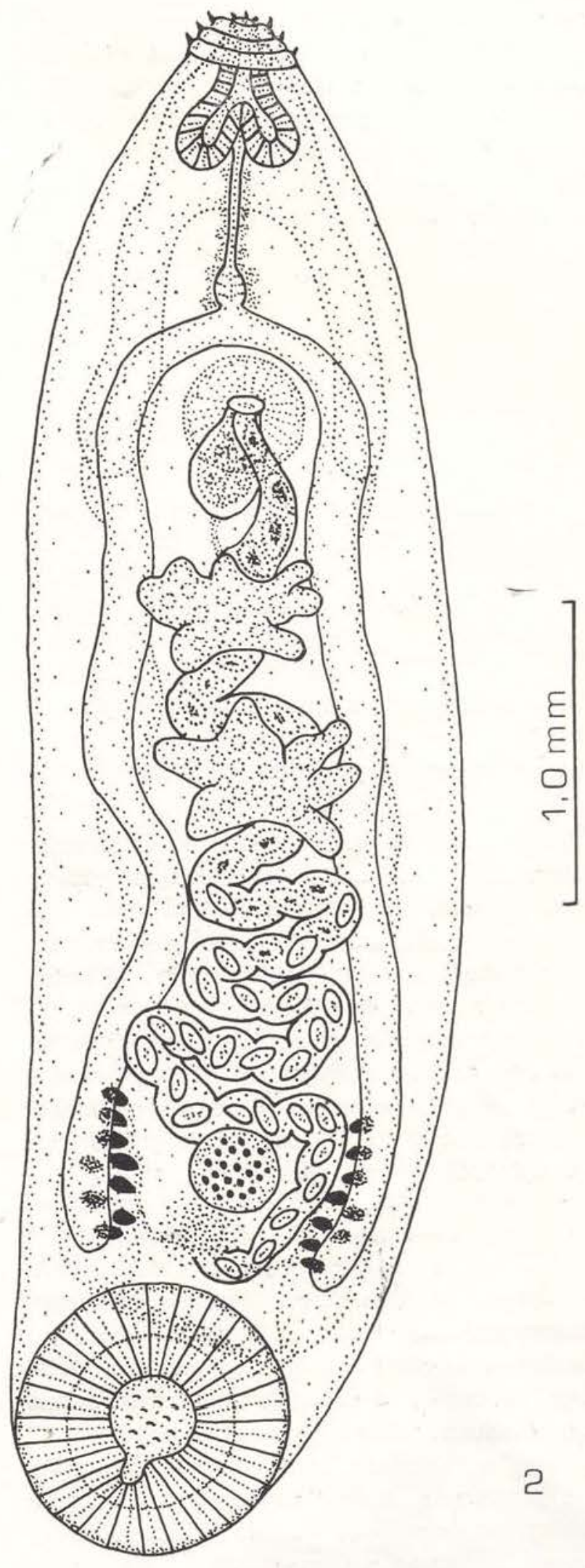

Fig. 2 - Dadaytrema oxycephala (Diesing, 1836) Travassos, 1931, vista ventral. 
terior à boca, Esôfago longo, envolvido com células glandulares. Pequeno bulbo esofagiano presente. Cecos medindo 0,13-0.18 $(0,14)$ de largura. Acetábulo com 0,56-0,90 $(0,65)$ de diâmetro e com uma depressão no bordo posterior do orifício. Testículos mais largos que compridos, medindo $0,43-0,86(0,50)$ de largura e $0,26-0,39(0,29)$ de comprimento. Bolsa genital variável em tamanho medindo $0,18-0,40$ $(0,28)$ de comprimento e $0,11-0,27 \quad(0,19)$ de largura. Ovos medem $60 \times 90 \mu$. Miracídios medem $85-96 \times 140-190 \mu$.

\section{DISCUSSÃo}

Existe na iiteratura muita confusão quanto à morfologia de $D$. oxycephala, advindo isso das dificultades em estudar formas tão grossos e cilíndricos. O presente estudo indicou a presença duma ventosa genital, duma depressão no bordo posterior do orifício do acetábulo e de quatro a cinco círculos de papilas atrás da boca. Nenhuma destas esrtuturas foram mencionadas na descrição mais recente (Travassos et al., 1969).

Uma bolsa do cirro é citado como característica do gênero tanto por Yamaguti (1958) como também por Travassos et al. (Ibid.). E lógico supor que um trematódeo que tem ventosa genital não precisa dum cirro. A bolsa que esta espécie tem é apenas para conter a vesícula seminal e, porém, deve ser considerada como uma bolsa genital. O que serve a função do cirro nesta espécie é, em realidade, a parede interior do pequeno átrio genital que é evaginavel.

\section{Dadaytremoides $\mathrm{n}$. gen}

Diagnose Genérica: Paramphistomidae, Dadaytrematinae; com as características da Família e Subfamília. Corpo achatado, mais largo na região equatorial, apontando-se nas extremidades. Cutícula lisa. Ventosa oral grande, arredondada, com divertículos grandes projetando atrás. Esôfago longo; bulbo esofagiano bem desenvolvido. Cecos largos, longos, atingindo o terço posterior do corpo. Acetábulo subterminal. Poro genital mediano atrás da bifurcação intestinal; pequena ventosa genital presente. Testículos fracamente lo- bados, diagonais, em meio terço do corpo. Ovário esférico, mediano, entre as terminações dos cecos. Glândulas vitelínicas constituídas por poucos folículos pequenos, dorsais as terminais dos cecos. Útero ocupando espaço intercecal entre ovário e testículos. Sistema circulatório típico da Subfamília presente. Vesicula excretora em forma de bolsa; poro dorsal. Ovovivíparos. Parasitas intestinais de peixes de água doce. Espécie tipo: $D$. gran. distomis n. sp.

\section{Dadaytremoides grandistomis n. sp.}

(Fig. 3)

Hospedeiros: Astyanax fasciatus (Cuvier). Chaetostomus leucomelas Eigenmann.

Habitat: Intestino.

Procedência: Rio Pance, Valle, Colômbia.

Holótipo: Instituto Nacional de Pesquisas da Amazônia (INPA)

Parátipos: INPA e Museu de Zoolngia da Universidade de São Paulo.

Diagnose específica (baseada em 7 exemplares): Com as características do gênero. Corpo medindo 1,9-4,0 $(2,6)$ de comprimento e $0,75-1,3$ de largura. Ventosa oral com 0,22-0,64 $(0,33)$ de diâmetro; divertículos medindo $0,14-0,22 \quad(0,16)$ de comprimento e $0,09-0,14(0,11)$ de largura. Boca terminal, circundada por pequenas papilas. Esôfago longo. Bulbc esofagiano medindo 0,09-0,14 $(0,11)$ de diâmetro. Cecos medindo 0,16-0,22 $(0,17)$ de largura. Acetábulo subterminal com $0,35-0,54$ $(0,46)$ de diâmetro. Testículos aproximadamente 0,19 de diâmetro. Bolsa genital pequena, com $0,14-0,23(0,15)$ de comprimento e $0,09-0,16(0,12)$ de largura. Ventosa genital medindo $0,12-0,18(0,16)$ de diâmetro. Glândulas vitelínicas constituídas por pequenas folículas com 0,04-0,09 diâmetro. Ovơs medem $36-45 \times 72-90 \mu$. Miracídios intrauterinos medem $41-62 \times 95-110 \mu$.

\section{DISCUSSÃO}

Dadaytremoides grandistomis $\mathrm{n}$. gen., $\mathrm{n}$. sp. aproxima-se mais do gênero Dadaytrema e da espécie tipo do mesmo, $D$. oxycephala, em ter divertículos orais grandes e uma distribuição semelhante dos órgãos internos. Ambos 
gêneros também mostram papilas circundando a boca. A nova forma distingue-se de $D$. oxycephala por ser menor, além de ter uma ventosa oral grande e esférica, testículos diagonais pouco lobados e um bulbo esofagiano bem desenvolvido. Além disso, $D$. grandistomis não tem papilas posteriormente à boca como é característica do gênero Dadaytrema. As duas espécies foram encontradas em hospedeiros diferentes e em sistemas de rios separados.

O nome do gênero indica a relação do mesmo com Dadaytrema e o nome específico refere ao tamanho maior da ventosa oral.

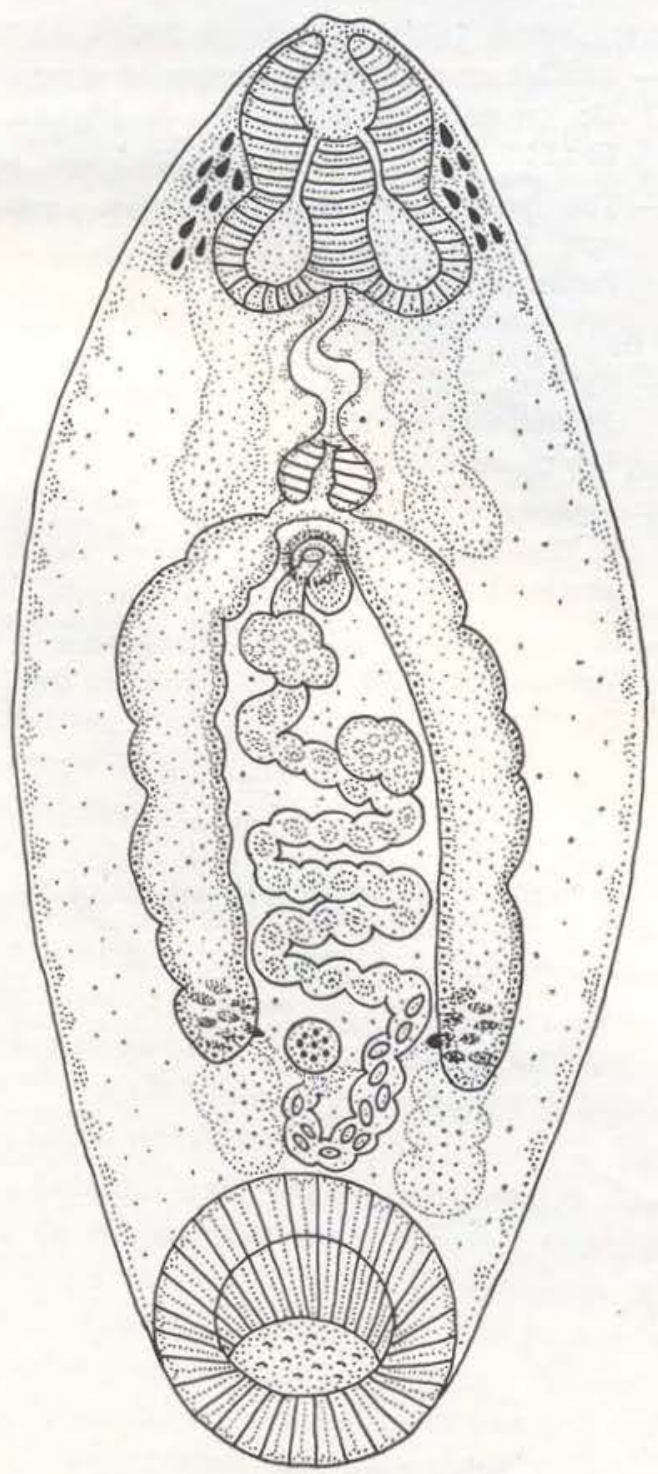

Fig. 3 -Dadaytremoides grandistomis $\mathrm{n}$, gen., $\mathrm{n}$. $\mathrm{sp}$. vista ventral.

\section{Conclusões}

Desde que foram descobertos no século passado, os representantes da familia Paramphistomidae tem apresentado problemas para estudar. Estas dificuldades devem-se principalmente à forma grande e grosso de muitos gêneros e à presença do sistema circulatório. É quase impossivel estudar a morfologia desses helmintos usando apenas os métodos clássicos que servem para os demais trema. tódeos, porque as precipitaçōes que formamse no interior dos vasos circulatórios escurecem os preparados. Como resultado disso, temus na literatura descrições de espécies e gêneros incompletas ou erradas.

No presente estudo, para superar estas dificuldades e visualizar melhor as estruturas, os trematódeos foram estudados vivos, bem como a fresco, clarificado com glicerina ou com fenol.

Com espécimes vivos foi possivel obser. var a saida de miracídios livres sem membra. na alguma, pelo poro genital em Dadaytrema oxycephala. Também se observou que ovos com casca estão produzidos no oötipo, mas duram pouco na passagem pelo útero. A casca do ovo desaparece deixando apenas um miracídio envolvido numa membrana. A membrana vai acomodando-se ao crescimento do miracidio e na parte distal do útero encontram-se miracídios já bem desenvolvidos e sem membrana. Todos os três gêneros reportados aqui tem a mesma formação de ovos e miracídios no útero $\mathrm{e}$, assim, devem ser considerados cumo ovovivíparos. O fato de ser ovovivíparo não foi reportado para $D$. oxycephala na descrição mais recente (Travassos et al., 1969) . Segundo parece, nenhum gênero de Paramphistomidae tem sido citado como ovovivíparo até agora, mas o fenômeno foi mencionado por Padilha (1978) para Zonocotylidae, Familia que pertence à mesma Superfamília.

C fato de produzir larvas vivas que são alimeniados dentro do útero, talvez por osmose, evidentemente limita a utilidade ou necessidade das glândulas vitelínicas. Nos gêneros apresentados aqui, essas glândulas são bem limitadas em distribuição e tamanho, enquanto nos gêneros que são ovíparos, por exemplo 
Catadiscus Cohn, 1904, e Nematophila Travassos, 1934, as glândulas de vitelo encontram-se bem desenvolvidas e amplamente distribuídas.

\section{SUMMARY}

Two new genera and species of Paramphistomidae Fischoeder, 1901, (Trematoda: Digenea) from the intestinal tracts of fish hosts were described, as follow: Colocladorchis ventrastomis $\mathrm{n}$. gen., $\mathrm{n}$. sr. from Prochilodus reticulatus and Dadaytremoides grandistomis n. gen., n. sp. from Astyanax fasciatus and Chaetostomus leucomelas. These species were collected near Cali, Valle, Colômbia.

Additionally, the genus Dadaytrema was redefined and D. oxycephala (Diesing. 1836) Travassos 1934, from Colossoma bidens, taken near Manaus, Amazonas, Brasil, was redescribed. All three species were found to be ovoviviparous.

\section{BIBLIOGRAFIA}

BENNETT, H.J.

1936 - The life history of Cotylophoron cotylophorum, a trematode from ruminants. Illinois Biol. Monogr., 14(9) : 1-119.

NÂSMARK, K.E.

1937 - A revision of the tremntode family $\mathrm{Pa}$ ramphistomidae. Zool. Bidrag., Uppsa. la. $16: 301-565$.

PADILHa, T.N.

1978 - Caracterização da Família Zonocotylidae com redescrição de Zonocotyle bicaecata Travassos, 1948 e descriçãj de um novo gênero (Trematoda, Digenea). Rev. Brasil. Biol, 38(2) : 415-426.
SKRJABIN, K.I.

1949 - Trematcdi Zhivotnikh i Cheloveka. (Tre matódeos dos animais e do homem). Akad. Nauk. S.S.S.R., $3: 1-495$.

THATCheR, V.E.

1978 - Quatro novas espécies de Haploporidae (Trematocaa: Digenea) de peixes de água doce de Colômbia com uma revisão do gênero Saccocoelioides Szidat, 1954. Acta Amazonica, 8(3) : 477-484.

Travassos, L.

1934 - Sinopse dos Paramphistomoidea. Mem. Inst. Oswaldo Cruz, 29(1) : 19-178.

Travassos, L.; Teixeira de Freitas, J.F. \& Kohn, A. 1969 - Trematódeos do Brasil. Mem. Inst, Os. waldo Cruz, $67: 1-886$.

WILLEY, C.H.

1930 - Studies on the lymph system of digenetic trematodes. J. Morph. \& Physiol., $50: 1-37$.

1933 - The lymph system of Zygocotyle luna tum (Trematoda, Paramphistomidae). Parasitol., 25 : 242-247.

Yamaguti, $\mathbf{S}$

1953 - Systema Helminthum, Part I., Digenetic Trematodes of Fishes. Pub. pelo autor. $405 \mathrm{p}$.

1958 - Systema Helminthum, Vol. I., Pts. 1 \& 2, The Digenetic Trematodes of Vertebrates. Intersci. Pub. Co. $1575 \mathrm{p}$.

1971 - Synopsis of Digenetic Trematodes of Vertebrates, Vols. I \& II. Keigaku Pub. Co. Tokio. $1774 \mathrm{p}$.

(Aceito para publicaçãc em 03/06/78) 